\title{
Recent Developments in Lattice Studies for Quarkonia
}

\author{
O. Kaczmarek \\ Fakultät für Physik, Universität Bielefeld, D-33615 Bielefeld, Germany
}

\begin{abstract}
After discussing results of dilepton rates and electrical conductivity obtained from continuum extrapolated results of light quark correlation functions in quenched QCD I will give a review on recent developments in lattice QCD studies for quarkonia in the quark gluon plasma. Recent progress in the extraction of spectral properties from lattice QCD calculations of hadronic correlation functions will be discussed. Besides medium modifications of bound states and their dissociation in the plasma I will focus on transport coefficients, like heavy quark diffusion constants extracted from different correlation functions on the lattice. Present limitations and future perspectives for studies of quarkonia and related transport coefficients on the lattice will be discussed. See also [1, 2, 3, 4] for recent overviews and related discussions of those topics.
\end{abstract}

Keywords: lattice QCD, quarkonium at finite temperature, heavy-ion collisions

\section{Dilepton rates and electrical conductivity}

A detailed knowledge of the spectral function of mesonic states at high temperatures is of fundamental importance for the understanding of the properties of the quark gluon plasma as well as for the analysis of this hot and dense medium in heavy ion collision experiments. In lattice QCD calculations the spectral function itself is not directly calculable but can be derived from hadronic correlation functions. In this section we will focus on the vector channel in the light quark sector before discussing heavy quark results in the remaining sections. The current-current correlation functions can be represented in terms of an integral over spectral functions, $\rho_{\mu v}(\omega, \vec{p}, T)$. We denote by $\rho_{i i}$ the sum over the three space-space components of the vector spectral function and also introduce the vector spectral function $\rho_{V} \equiv \rho_{00}+\rho_{i i}$. With this we obtain the corresponding correlation functions,

$$
G_{H}(\tau, \vec{p}, T)=\int_{0}^{\infty} \frac{\mathrm{d} \omega}{2 \pi} \rho_{H}(\omega, \vec{p}, T) \frac{\cosh (\omega(\tau-1 / 2 T))}{\sinh (\omega / 2 T)} \quad, \quad H=00, i i, V .
$$

The vector spectral function is directly related to the thermal production rate of dilepton pairs with squared invariant mass $\omega^{2}-\vec{p}^{2}$

$$
\frac{\mathrm{d} N_{l^{+} l^{-}}}{\mathrm{d} \omega \mathrm{d}^{3} p}=C_{e m} \frac{\alpha_{e m}^{2}}{6 \pi^{3}} \frac{\rho_{V}(\omega, \vec{p}, T)}{\left(\omega^{2}-\vec{p}^{2}\right)\left(\mathrm{e}^{\omega / T}-1\right)},
$$

where $\alpha_{e m}$ is the electromagnetic fine structure constant. This expression is correct to leading order in the electromagnetic interaction but contains the full QCD information once the vector spectral function is known. (see also [2]). In the following only the $\vec{p}=0$ case will be discussed. 


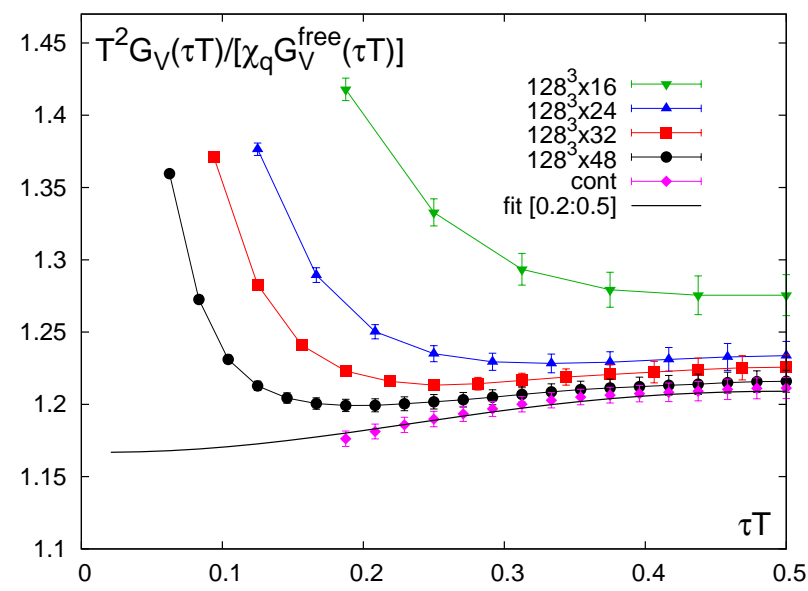

Figure 1: Lattice QCD results together with the continuum extrapolation of the vector correlation function at $1.46 T_{c}$ from [5].

In [5] a controlled continuum extrapolation of the vector correlation function calculated in quenched QCD at $T \simeq 1.45 T_{c}$ was performed for the first time. Those lattice results of [5] together with the result in the continuum limit are shown in Fig. 1 .

The vector correlation function in the continuum was used to fit to a phenomenological inspired Ansatz for spectral function,

$$
\begin{aligned}
\rho_{00}(\omega) & =-2 \pi \chi_{q} \omega \delta(\omega), \\
\rho_{i i}(\omega) & =2 \chi_{q} c_{B W} \frac{\omega \Gamma / 2}{\omega^{2}+(\Gamma / 2)^{2}}+\frac{3}{2 \pi}(1+k) \omega^{2} \tanh (\omega / 4 T),
\end{aligned}
$$

which contains a Breit-Wigner contribution at small frequencies that gives the correct $\omega$-dependence in the small frequency limit to determine the electrical conductivity and a correction parameter $k(T)$ that parameterizes deviations from a free spectral function at large energies. The result of this fit shown in Fig. 1 demonstrates that already this minimal ansatz provides a good description of current numerical results for the vector correlation function.
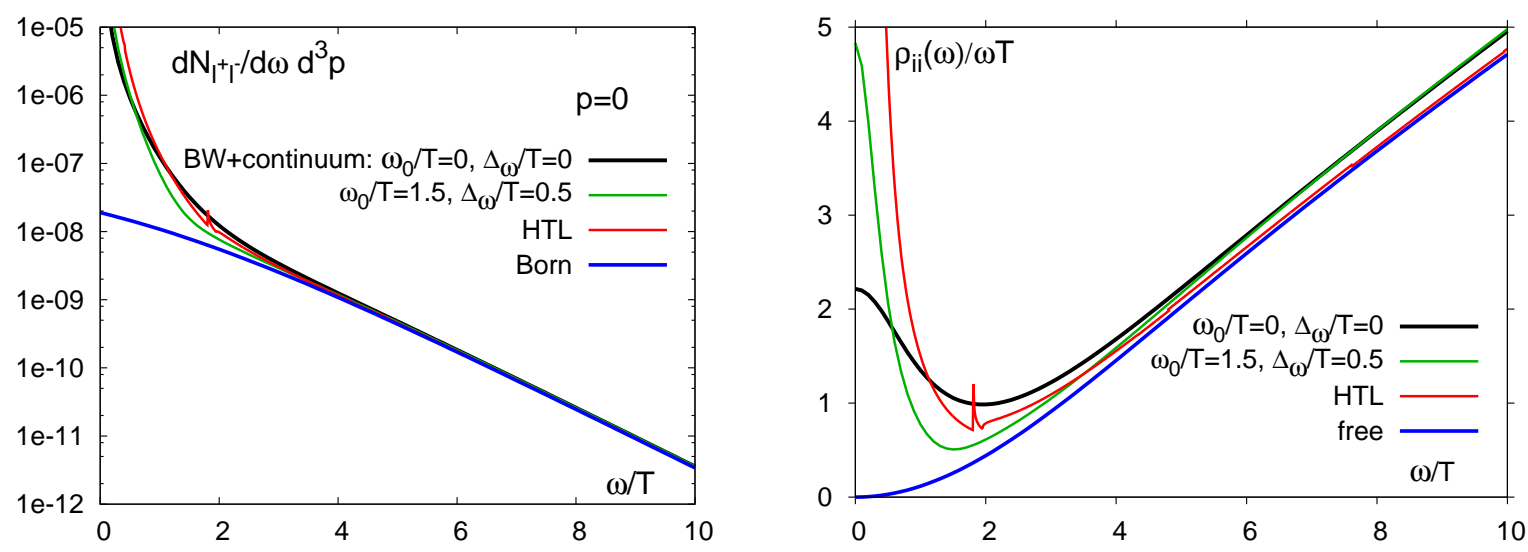

Figure 2: Thermal dilepton rate in 2-flavor QCD (left). The HTL curve is for a thermal quark mass $m_{T} / T=1$ and the Born rate is obtained by using the free spectral function. The right hand part of the figure shows the spectral functions that entered the calculation of the dilepton rate. (see text and [5] for details).

Using Eq. (2) the continuum estimate for the dilepton rate at $T \simeq 1.45 T_{c}$ in Fig. 2(left) are obtained. They merge with the Born-rate at $\omega \gtrsim 4$ and are well above this rate for lower energies, still staying below the HTL result at 

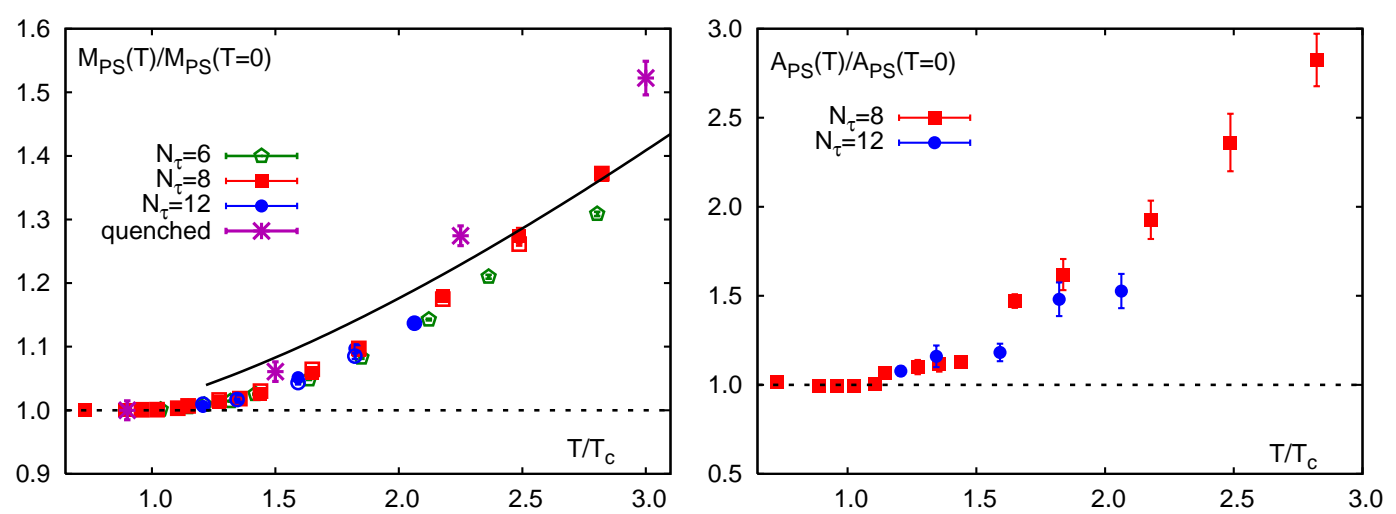

Figure 3: Pseudo-scalar screening mass (a) and amplitude (b) divided by the corresponding zero temperature values as function of the temperature. The solid black line is the free quark result. The plots are from [9].

small $\omega$. In contrast to the HTL result, the limit $\omega \rightarrow 0$ is well behaved (see Fig. 2(right)). In this limit the spatial components of the vector spectral function determine the electrical conductivity

$$
\frac{\sigma}{T}=\frac{C_{e m}}{6} \lim _{\omega \rightarrow 0} \frac{\rho_{i i}(\omega)}{\omega T} .
$$

The vector spectral function shown in Fig. 2 at vanishing energy leads to an estimate of the electrical conductivity

$$
1 / 3 \lesssim \frac{1}{C_{e m}} \frac{\sigma}{T} \lesssim 1 \quad \text { at } \quad T \simeq 1.45 T_{c}
$$

Although only a lower and upper limit was estimated, the result demonstrates that transport coefficients can be reliably extracted from continuum extrapolated lattice QCD correlation functions which in this respect improves on earlier results [6, 7]. See [5] for more details and [8] for a recent study using a new technique to extract the spectral function in this limit.

The vector spectral function at light-like 4-momentum yields the photon emission rate of a thermal medium,

$$
\omega \frac{\mathrm{d} R_{\gamma}}{\mathrm{d}^{3} p}=C_{e m} \frac{\alpha_{e m}}{4 \pi^{2}} \frac{\rho_{V}(\omega=|\vec{p}|, T)}{\mathrm{e}^{\omega / T}-1} .
$$

The emission rate of soft photons, thus can be related to the electrical conductivity,

$$
\lim _{\omega \rightarrow 0} \omega \frac{\mathrm{d} R_{\gamma}}{\mathrm{d}^{3} p}=\frac{3}{2 \pi^{2}} \sigma(T) T \alpha_{e m} .
$$

Together with Eq. (7) this yields for the zero energy limit of the thermal photon rate,

$$
\lim _{\omega \rightarrow 0} \omega \frac{\mathrm{d} R_{\gamma}}{\mathrm{d}^{3} p}=(0.0004-0.0013) T_{c}^{2} \simeq(1-3) \cdot 10^{-5} \mathrm{GeV}^{2} \quad \text { at } \quad T \simeq 1.45 T_{c} .
$$

Results of the vector correlation function at non-zero momentum will be used in the future to estimate $p \neq 0$ dilepton rates and the finite energy contribution of photons in the QGP.

\section{Charmonium screening masses}

High precision data at a large number of Euclidean time separations are needed to extract spectral properties from temporal correlation function. An alternative approach that may provide additional information on the in-medium modifications of spectral functions and medium effects of mesonic states are spatial correlation functions,

$$
G(z, T)=\int d x d y \int_{0}^{1 / T} d \tau\langle J(x, y, z, \tau) J(0,0,0,0)\rangle,
$$



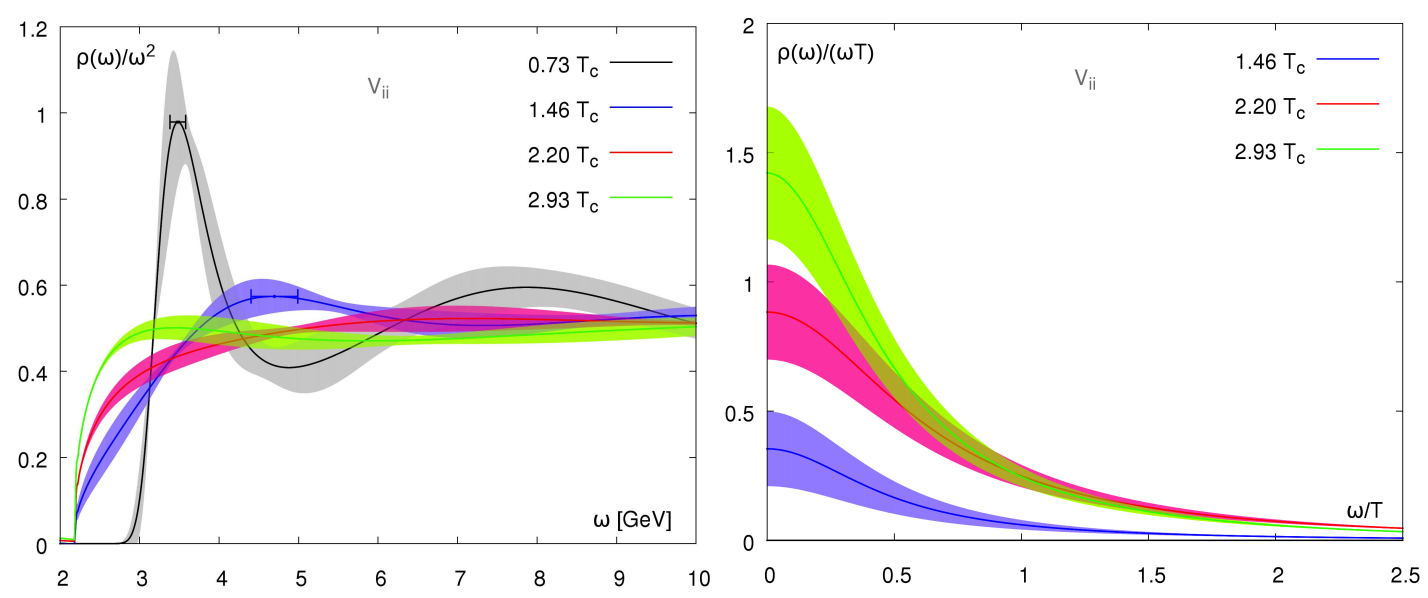

Figure 4: Results for the charmonium spectral functions in the vector channel. Shaded areas are statistical errors from Jackknife analyses. Horizontal error bars at the first peaks of spectral functions at $0.73 T_{c}$ an $1.4 T_{c}$ indicate the statistical uncertainties of the peak location obtained from the Jackknife analyses. (see [10] for details).

which are related to the momentum dependent spectral function through

$$
G(z, T)=\int_{0}^{\infty} \frac{d \omega}{2 \pi} \frac{2}{\omega} \int_{-\infty}^{\infty} \frac{d p_{z}}{2 \pi} e^{i p_{z} z} \rho\left(\omega, p_{z}, T\right) .
$$

At large distance, $z T>1$, where medium effects are expected to be largest, $G(z, T)$ decays exponentially allowing to define a screening mass $M_{s c r}$. If bound states appear in the spectral function, the screening mass is determined by the mass $M$ of the lowest lying meson state. On the other hand for unbound quark antiquark pairs at high temperature, $M_{s c r}$ is given by the lowest non-vanishing Matsubara frequency $2 \sqrt{(\pi T)^{2}+m_{c}^{2}}$ where $m_{c}$ is the quark mass. Those two limits and the transition between them may serve a an indication for significant modifications and dissociation of heavy quark meson states.

In Fig. 3 the results for the charmonium screening masses calculated for a dynamical $2+1$ flavor lattice QCD from [9] are shown, normalized to the zero temperature value. The change in the temperature dependence becomes apparent around 1.5 $T_{c}$ where deviations from the zero temperature limit become large and the behavior becomes compatible with that of unbound $c \bar{c}$ pairs. This is an indication that charmonium states melt around this temperature which is a consistent picture with the findings from the study of temporal charmonium correlators in quenched QCD [10] that will be discussed in the following section.

\section{Charmonium spectral functions and heavy quark diffusion}

The properties of charmonium states at finite temperature were studied in [10] in quenched lattice QCD on large and fine isotropic lattices. Although no continuum extrapolation was performed, the results for lattice sizes of $128^{3} \times N_{\tau}$ for $N_{\tau}$ between 16 and 96 and lattice spacing $a$ down to $0.01 \mathrm{fm}$ are already close to the continuum and cut-off effects in the spectral function are well separated from the physically interesting energy regions, as $a m_{c}$ is much smaller than one. In [10] a detailed analysis of statistical and systematic uncertainties and default model dependencies in the maximum entropy method (MEM) used to determine the spectral functions from the lattice calculations of the charmonium correlation functions was performed.

The results of this study, shown in Fig. 4 for the vector channel, suggest that charmonium states are dissociated already at $1.46 T_{c}$. While at $0.73 T_{c}$ stable and reliable ground state peaks are found, at the higher temperatures in this study no signals for bound state contributions but rather a threshold enhancement are seen.

The slope of the vector spectral function in the $\omega \rightarrow 0$ limit again determines a transport coefficient, the heavy 


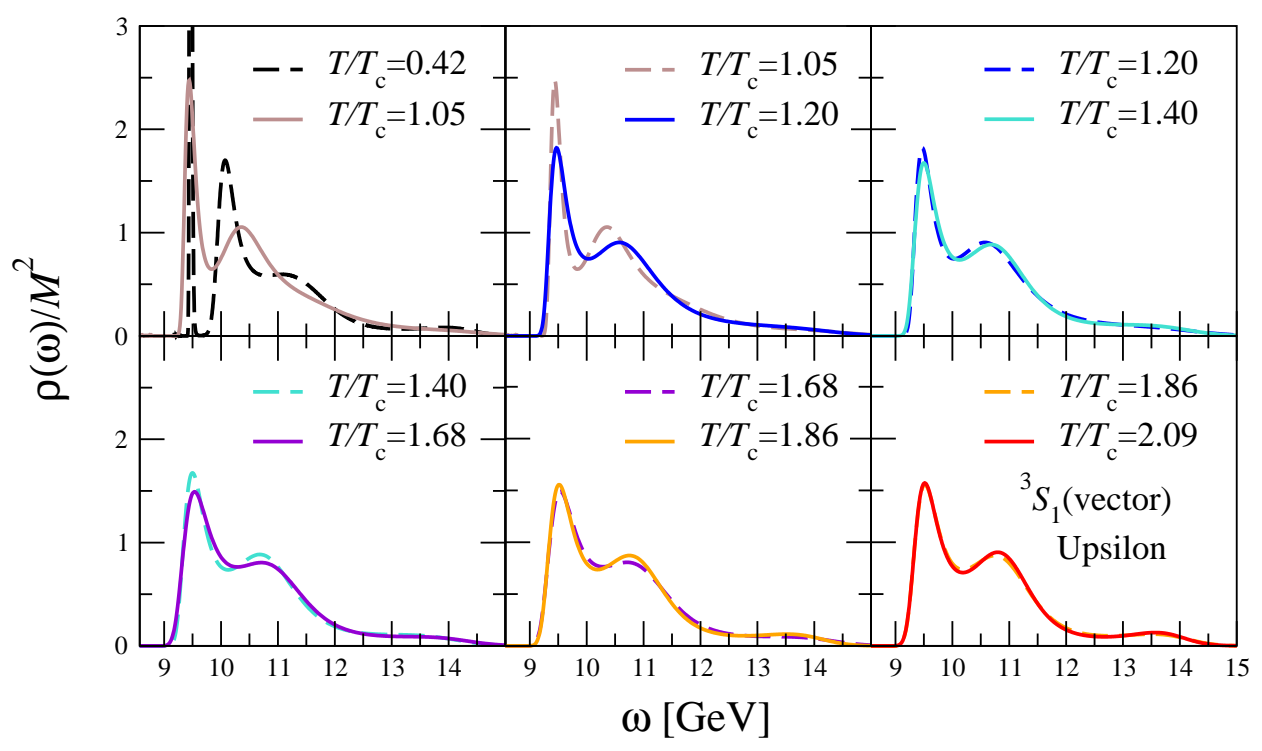

Figure 5: Spectral functions $\rho(\omega)$ in the vector channel $(\Upsilon)$, normalized with the heavy quark mass. This plot is from [11].

quark diffusion constant $D$,

$$
D=\frac{1}{6 \chi^{00}} \lim _{\omega \rightarrow 0} \sum_{i=1}^{3} \frac{\rho_{i i}^{V}(\omega, \vec{p}=0, T)}{\omega}
$$

where $\chi^{00}$ is the quark number susceptibility. While compatible with zero below $T_{c}$, almost temperature independent values of $2 \pi T D$ around two at $T=1.46,2.20$ and 2.93 were obtained in [10] (see Fig. 77).

This is a further indication of strong medium effects and $J / \Psi$ being melted at $1.46 T_{c}$ and higher temperatures. Together with the results for the charmonium screening masses of the previous section this gives a consistent picture and shows that the interesting temperature region for medium effects and the study for dissociation of charmonium is between $T_{c}$ and $1.5 T_{c}$. Future studies at temperatures closer to $T_{c}$ using continuum extrapolated correlation functions may allow a more detailed determination of the dissociation patterns of the different charmonium states in the quark gluon plasma.

\section{Bottomonium from lattice NRQCD}

At the energies probed at the LHC bottomonium spectroscopy becomes an important tool for the analysis of the produced hot medium. It may offer a cleaner probe compared to charmonium as feed-down effects from heavier quarkonium states are missing and regeneration from the thermal medium will be negligible. Furthermore the dissociation temperatures are far higher than for charmonium and therefore well separated from the hadronization stage. First results from the CMS experiment at LHC show a suppression of the higher excited states $\Upsilon(2 S+3 S)$ [12] and already indicate the importance of bottomonium in this respect. Therefore a detailed knowledge on the behavior and dissociation temperatures of bottomonium states is of fundamental importance.

For bottom quarks the heavy quark mass scale can be integrated out in terms of an effective field theory, nonrelativistic QCD (NRQCD). In a lattice discretization of this effective theory where the physics above the scale $m_{b}$ is encoded in the perturbatively determined coefficients of the NRQCD Lagrangian, the spatial lattice spacing acts as a short distance cut-off and must satisfy $m_{b} a_{s} \gtrsim 1[11,13,14]$. The drawback of this is that no continuum limit is possible in lattice NRQCD and furthermore that cut-off effects are present in the spectral function already in the energy region relevant for bottomonium excited states as the cut-off effects and also the accessible energy region is determined by the spatial lattice spacing. 

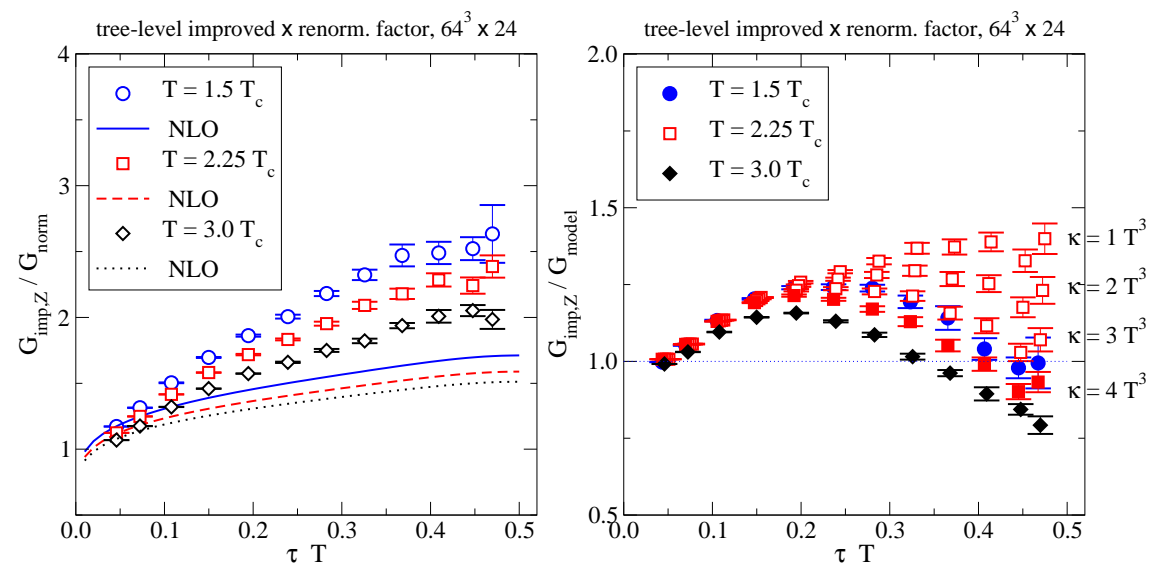

Figure 6: Left: Comparison of lattice with the NLO weak-coupling expansion [15]. Right: Like on the left but normalized to the model of eq.15 Closed symbols correspond to $\kappa=4 T^{3}$, open ones to $T=2.25 T_{\mathrm{c}}$ (see [16] for details).

In contrast to a computationally expensive inversion of the fermion matrix, NRQCD propagators are calculated as an initial value problem. Information on transport properties are exponentially suppressed which is a further consequence of the quark mass scale acting as a low energy cut-off at $2 m_{b}$. Nevertheless this facilitates the extraction of spectral properties in the bound state region as no constant contribution appear in the NRQCD correlation functions.

Despite the limitations of the method, the results of [11] demonstrate the benefits of lattice NRQCD. Their results for the spectral function in the vector channel $(\Upsilon)$ are shown in Fig. 5. It suggests that the ground state survives up to the highest temperature analyzed in this work and indicates the melting of higher excited states at temperatures above $1.4 T_{c}$. See [11] for a detailed discussion of the results.

Especially for the behavior of the excited states a detailed analysis of the systematic uncertainties of this approach is still needed, especially to understand the cut-off effects and default model dependencies of the maximum entropy method used to extract the spectral functions. In addition lattice NRQCD results will be useful for potential model studies of quarkonium systems and will give additional input for future full relativistic lattice QCD calculations of bottomonium states.

\section{Heavy quark momentum diffusion coefficient}

Effective field theory methods are also important to derive operators to extract transport coefficients from lattice QCD. In the large quark mass limit Heavy Quark Effective Theory (HQET) can be used to derive a purely gluonic operator which is related to the "momentum diffusion coefficient" in the low-frequency limit,

$$
\kappa=\lim _{\omega \rightarrow 0} \frac{2 T \rho_{\mathrm{E}}(\omega)}{\omega} .
$$

Heavy quarks carry a color charge and are therefore subject to a colored Lorentz force. Like with other transport coefficients the corresponding "low-energy constants" are easiest to define at vanishing three-momentum when the Lorentz-force is proportional to the electric field strength. This leads to a "color-electric correlator" [17, 18],

$$
G_{\mathrm{E}}(\tau) \equiv-\frac{1}{3} \sum_{i=1}^{3} \frac{\left\langle\operatorname{Re} \operatorname{Tr}\left[U\left(\frac{1}{T} ; \tau\right) g E_{i}(\tau, \overrightarrow{0}) U(\tau ; 0) g E_{i}(0, \overrightarrow{0})\right]\right\rangle}{\left\langle\operatorname{Re} \operatorname{Tr}\left[U\left(\frac{1}{T} ; 0\right)\right]\right\rangle},
$$

where $g E_{i}$ denotes the color-electric field, $T$ the temperature, and $U\left(\tau_{2} ; \tau_{1}\right)$ a Wilson line in Euclidean time direction. If the corresponding spectral function, $\rho_{\mathrm{E}}$, can be extracted [19], then the "momentum diffusion coefficient", often denoted by $\kappa$, can be obtained from Eq. (13). According to non-relativistic linear response relations (valid for $M \gg \pi T$, where $M$ stands for a heavy quark pole mass) the corresponding "diffusion coefficient" is given by $D=2 T^{2} / \kappa$. 


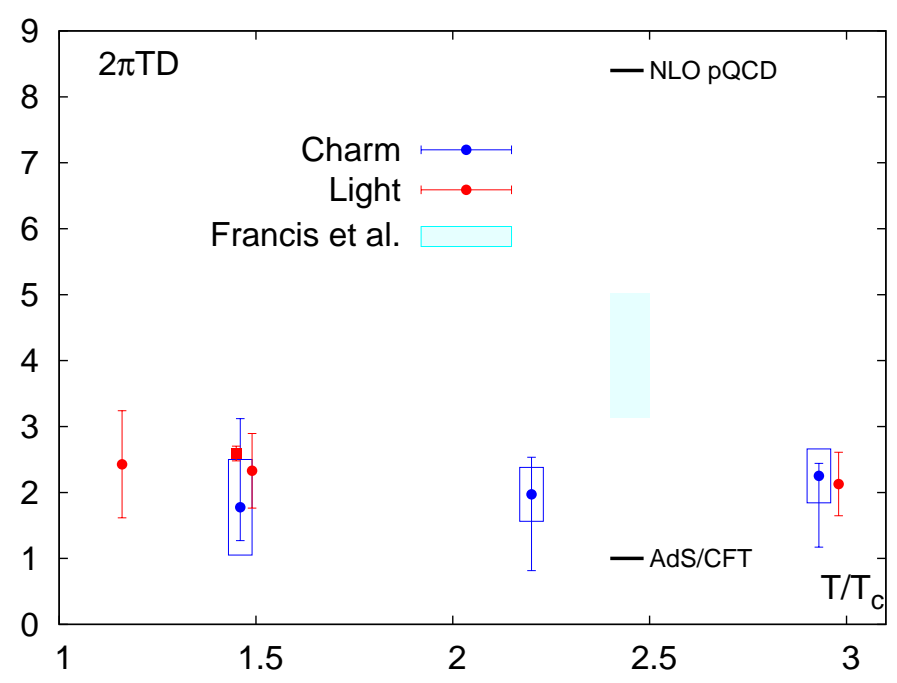

Figure 7: Comparison of various estimates for the heavy quark diffusion constant (see the text for more details).

Due to the gluonic nature of the operator Eq. (14) large fluctuations lead to weak signal/noise ratio. Using clever improvement algorithms in addition with tree-level improvement to reduce cut-off effects and a perturbative renormalization of the operator leads to results that show almost no lattice artifacts.

In Fig. 6(left) the results of [16] are shown in comparison with a NLO weak-coupling expansion, both normalized by the leading order [18] correlation function. To extract the diffusion coefficient we use a model for the spectral function

$$
\rho_{\text {model }}(\omega) \equiv \max \left\{\rho_{\mathrm{NLO}}(\omega), \frac{\omega \kappa}{2 T}\right\}
$$

with the free parameter $\kappa$ representing directly the momentum diffusion coefficient according to Eq. (13), and the corresponding Euclidean correlator computed from

$$
G_{\text {model }}(\tau) \equiv \int_{0}^{\infty} \frac{\mathrm{d} \omega}{\pi} \rho_{\text {model }}(\omega) \frac{\cosh \left(\left(\frac{1}{2}-\tau T\right) \frac{\omega}{T}\right)}{\sinh \left(\frac{\omega}{2 T}\right)}
$$

Results for the ratio of the lattice data and the model correlation function for different values of $\kappa$ are shown in Fig. 6 and allow for an estimate for the momentum diffusion constant at the three temperatures analyzed. Converting those estimates to the usual diffusion coefficient $D=2 T^{2} / \kappa$ one obtains $D T \sim(0.5 \cdots 0.8)$ which is slightly larger than the values obtained for the charm case [10] (see section 3) and on this qualitative level consistent with a similar analysis in [20].

Although the values for $\kappa$ and $D T$ are to be understood as a first rough estimate, it indicates that a consistent picture appears to emerge. Improving the lattice data for the correlation function and performing the continuum limit, which is possible in this approach, will allow to test improved models for the spectral function and use methods like the maximum entropy method or other sophisticated methods to perform the analytic continuation like the one proposed and tested in [21, 8].

\section{Conclusions and outlook}

Lattice QCD calculations have proven to provide important information on the in-medium modifications of hadronic states for light up to bottom quarks. High quality results and sophisticated methods to determine spectral properties from lattice correlation functions allow a reliable estimate of transport coefficients. This is accompanied by effective field theory methods used in the heavy quark mass limit. Especially for the analysis of bottomonium and even more 
for the determination of transport coefficients from lattice correlation functions additional information can be gained from this combination.

Although most results are still obtained in the quenched limit or for rather large light quark masses, a consistent picture of medium modifications and the dissociation of quarkonia as well as of transport coefficients needed to describe the thermal medium and the behavior of heavy quarks in this medium emerges.

For future charmonium studies the interesting temperature region between $T_{c}$ and $1.5 T_{c}$ shall be explored in the future as the recent results indicate that charmonium is melted already above those temperatures. At least for the bottomonium ground states higher temperatures seem to be relevant and full relativistic lattice calculation are necessary to confirm the findings of the exploratory NRQCD study in this sector.

In Fig. 7 we compare the values for the diffusion coefficients obtained from the analysis of the charmonium spectral function in quenched QCD [10] and the heavy quark momentum diffusion coefficient in the large quark mass limit [16] (light blue band). On this qualitative level all results are compatible with estimates derived from the heavy flavor $R_{A A}$ and $v_{w}$ PHENIX data [22, 23]. See also [24] for a determination of the diffusion constant using heavy quark free energies in a T-matrix approach and [25] for a recent discussion of heavy quark diffusion constants. Just for comparison we also show the result obtained from the electrical conductivity in the light quark sector [5, 26].

The results of section 4 and 5 show the opportunity and applicability of effective field theory methods and operators derived from it for the study of heavy quark spectral and transport properties in lattice QCD calculations. Despite their limitations they will allow to constrain models, e.g. for potential model calculations [3, 27], and contribute additional information and input needed in full relativistic lattice QCD calculations in the future especially for the difficult task of extracting spectral properties from hadronic correlation functions.

In a similar way as for the light quark case discussed in section 1, an extrapolation of the correlation functions to the continuum will be required in the heavy quark sector to remove any lattice discretization effects. Although at the moment only possible in quenched QCD this will allow a more quantitative determination of spectral and transport properties for quarkonium systems. This will lay the foundation for the analysis of quarkonium correlation functions in full QCD with realistic light quark mass becoming available in the coming years at least for moderate temporal lattice extensions and will allow to determine the effects of light quark masses on spectral properties of heavy mesons.

\section{References}

[1] B. Muller, these proceedings, arXiv: 1207.7302

[2] C. Gale, these proceedings, arXiv: 1208.2289

[3] A. Rothkopf, these proceedings, arXiv:1207.5486

[4] H.-T. Ding, arXiv: 1207.5187

[5] H.-T. Ding, A. Francis, O. Kaczmarek, F. Karsch, E. Laermann, et al., Phys.Rev. D83 (2011) 034504.

[6] S. Gupta, Phys.Lett. B597 (2004) 57-62.

[7] G. Aarts, C. Allton, J. Foley, S. Hands, S. Kim, Phys.Rev.Lett. 99 (2007) 022002.

[8] Y. Burnier, M. Laine, Eur.Phys.J. C72 (2012) 1902.

[9] F. Karsch, E. Laermann, S. Mukherjee, P. Petreczky, Phys.Rev. D85 (2012) 114501.

[10] H.-T. Ding, A. Francis, O. Kaczmarek, F. Karsch, H. Satz, et al., Phys.Rev. D86 (2012) 014509.

[11] G. Aarts, C. Allton, S. Kim, M. Lombardo, M. Oktay, et al., JHEP 1111 (2011) 103.

[12] R. Reed, J.Phys.G G38 (2011) 124185.

[13] G. Aarts, S. Kim, M. Lombardo, M. Oktay, S. Ryan, et al., Phys.Rev.Lett. 106 (2011) 061602.

[14] J. Fingberg, Phys.Lett. B424 (1998) 343-354.

[15] Y. Burnier, M. Laine, J. Langelage, L. Mether, JHEP 1008 (2010) 094.

[16] A. Francis, O. Kaczmarek, M. Laine, J. Langelage, PoS LATTICE2011 (2011) 202.

[17] J. Casalderrey-Solana, D. Teaney, Phys.Rev. D74 (2006) 085012.

[18] S. Caron-Huot, M. Laine, G. D. Moore, JHEP 0904 (2009) 053.

[19] H. B. Meyer, Eur.Phys.J. A47 (2011) 86.

[20] D. Banerjee, S. Datta, R. Gavai, P. Majumdar, Phys.Rev. D85 (2012) 014510.

[21] Y. Burnier, M. Laine, L. Mether, Eur.Phys.J. C71 (2011) 1619.

[22] A. Adare, et al., Phys.Rev. C84 (2011) 044905.

[23] A. Adare, et al., Phys.Rev.Lett. 98 (2007) 172301

[24] F. Riek, R. Rapp, Phys.Rev. C82 (2010) 035201.

[25] M. He, R. J. Fries, R. Rapp, arXiv:1204.4442

[26] A. Francis, O. Kaczmarek, Prog.Part.Nucl.Phys. 67 (2012) 212-217.

[27] Y. Burnier, A. Rothkopf, arXiv: 1208.1899 